Article

\title{
Aflatoxin Biosynthesis Is a Novel Source of Reactive Oxygen Species-A Potential Redox Signal to Initiate Resistance to Oxidative Stress?
}

\author{
Ludmila V. Roze ${ }^{1,2}$, Maris Laivenieks ${ }^{3}$, Sung-Yong Hong ${ }^{1}$, Josephine Wee ${ }^{1}$, Shu-Shyan Wong ${ }^{1}$, \\ Benjamin Vanos ${ }^{1}$, Deena Awad ${ }^{1}$, Kenneth C. Ehrlich ${ }^{4}$ and John E. Linz ${ }^{1,3,5, *}$
}

1 Department of Food Science and Human Nutrition, Michigan State University (MSU), East Lansing, MI 48824, USA; E-Mails: roze@msu.edu (L.V.R.); lunohong@yahoo.co.kr (S.-Y.H.); weejosep@msu.edu (J.W.); wongs94@yahoo.com (S.-S.W.); vanosben@msu.edu (B.V.); awaddeen@msu.edu (D.A.)

2 Department of Plant Biology, Michigan State University (MSU), East Lansing, MI 48824, USA

3 Department of Microbiology and Molecular Genetics, Michigan State University (MSU), East Lansing, MI 48824, USA; E-Mail: laivenie@msu.edu

4 Southern Regional Research Center, Agricultural Research Service, USDA, New Orleans, LA 70124, USA; E-Mail: ehrlich8@gmail.com

5 Center for Integrative Toxicology, Michigan State University (MSU), East Lansing, MI 48824, USA

* Author to whom correspondence should be addressed; E-Mail: jlinz@msu.edu; Tel.: +1-517-355-8474; Fax: +1-517-353-8963.

Academic Editor: Jiujiang Yu

Received: 5 February 2015 / Accepted: 17 April 2015 / Published: 28 April 2015

\begin{abstract}
Aflatoxin biosynthesis in the filamentous fungus Aspergillus parasiticus involves a minimum of 21 enzymes, encoded by genes located in a $70 \mathrm{~kb}$ gene cluster. For aflatoxin biosynthesis to be completed, the required enzymes must be transported to specialized early and late endosomes called aflatoxisomes. Of particular significance, seven aflatoxin biosynthetic enzymes are $\mathrm{P} 450$ /monooxygenases which catalyze reactions that can produce reactive oxygen species (ROS) as byproducts. Thus, oxidative reactions in the aflatoxin biosynthetic pathway could potentially be an additional source of intracellular ROS. The present work explores the hypothesis that the aflatoxin biosynthetic pathway generates ROS (designated as "secondary" ROS) in endosomes and that secondary ROS possess a signaling function. We used specific dyes that stain ROS in live cells and demonstrated that intracellular ROS levels correlate with the levels of aflatoxin synthesized. Moreover, feeding
\end{abstract}


protoplasts with precursors of aflatoxin resulted in the increase in ROS generation. These data support the hypothesis. Our findings also suggest that secondary ROS may fulfill, at least in part, an important mechanistic role in increased tolerance to oxidative stress in germinating spores (seven-hour germlings) and in regulation of fungal development.

Keywords: Aspergillus parasiticus; oxidative stress; secondary metabolism; aflatoxin; endosomes; redox signaling

\section{Introduction}

In fungi redox signaling with the involvement of reactive oxygen species (ROS) has been implicated in a large number of processes including differentiation, asexual and sexual reproduction, sclerotia development, and fungal-plant interaction [1-11]. ROS, and in particular superoxide radical, have also been reported to control secondary metabolism [12]. Intracellular formation of ROS involves mainly mitochondrial oxidative phosphorylation, and reactions catalyzed by cell membrane, endosome NADPH oxidases (NOX), and enzymes for beta-oxidation of lipids in mitochondria and peroxisomes [3]. Oxidation reactions performed by monooxygenases/cytochrome P450 (CYP) isozymes, nitric oxide synthases, and xanthine oxidases also contribute to production of intracellular ROS [13-20]. Several studies provided evidence that CYP enzymes generate superoxide and hydrogen peroxide as intermediate compounds, and that these ROS can cause apoptosis and other cell pathologies [14,19,21-23].

Secondary metabolite pathways that are present in fungi and plants comprise a large number of CYPs and monooxygenases that carry out oxidative transformation of the intermediates in secondary metabolism. Consequently, these oxidative reactions could potentially be an additional uncharacterized source of intracellular ROS.

The filamentous fungus Aspergillus parasiticus is a focus of intense research effort due to its ability to synthesize the secondary metabolite and the potent carcinogen aflatoxin during growth on susceptible host plants. Aflatoxin biosynthesis occurs in endosomes/aflatoxisomes within the fungal cell; these compartments also enable storage and export of aflatoxin to the cell exterior [24]. The twenty-seven genes that encode aflatoxin biosynthetic enzymes are clustered; the entire aflatoxin biosynthetic pathway includes at least 21 enzymatic reactions [25]. Although aflatoxin biosynthesis is regulated by intracellular ROS of different origins $[10,12]$, the aflatoxin biosynthetic pathway itself may serve as a source of ROS due to involvement of P450 enzymes. Indirect support for the idea of ROS production by aflatoxin biosynthetic pathway was provided by studies showing that an aflatoxin producing A. parasiticus strain generated significantly higher quantities of intracellular ROS than an aflatoxin non-producer [26]. Moreover, the same study demonstrated that genetic block in the aflatoxin biosynthetic pathway at an early, middle, or late step, correspondingly affected quantities of detected ROS.

In the current study we test the hypothesis that the aflatoxin biosynthetic pathway generates ROS which we designate "secondary ROS". Since biosynthetic steps of aflatoxin biosynthesis are compartmentalized within endosomes/aflatoxisomes, these sub-cellular organelles could represent loci where secondary ROS is generated in association with distinct biosynthetic steps. 
Using staining techniques which detect ROS in live $A$. parasiticus mutants that generate different quantities of aflatoxin, and feeding protoplasts with intermediates of aflatoxin biosynthetic pathway, we present data that support the hypothesis. The data suggest that the secondary ROS initiate redox signaling from endosomes/aflatoxisomes and that one downstream target of the signal is increased tolerance to oxidative stress in the next generation of germlings. This study contributes to our understanding of the role of secondary metabolism in fungal biology and helps to answer the question "why are secondary metabolites made?"

\section{Results}

\subsection{Enzymes in Aflatoxin Biosynthesis Are a Potential Source of Secondary ROS}

Table 1 lists the enzymes involved in aflatoxin biosynthesis that serve as potential sources of secondary ROS. At least seven enzymes encoded by cypA (aflU), avnA (aflG), avfA (aflI), verA (aflN), $\operatorname{ordA}($ aflQ), verB (aflL), and $\operatorname{cyp} X$ (aflV), belong to a superfamily of P450 monooxygenases that is capable of generating superoxide radicals. Among enzymes that participate in aflatoxin biosynthesis are two monooxygenases, aflX ( ordB) and aflW (moxY). These enzymes are involved in early (hypC (38), cyp $A)$, middle (verA, avnA), and late steps ( $\operatorname{ord} A, \operatorname{cyp} A, \operatorname{mox} Y, \operatorname{ord} A)$ in the biosynthetic pathway.

Table 1. Enzymes and reactions that are potential sources of "secondary" reactive oxygen species (ROS) in aflatoxin biosynthetic pathway.

\begin{tabular}{|c|c|c|c|}
\hline $\begin{array}{c}\text { Gene ID in } A . \text { flavus } \\
\text { genome database }\end{array}$ & $\begin{array}{c}\text { Gene symbol } \\
\text { (old gene symbol) }\end{array}$ & Gene/enzyme name & Reaction, reference \\
\hline AFLA_139140 & aflYa (nadA) & NADH oxidase & formation of $\mathrm{AFG}_{1}$ from $\mathrm{HOMST}$ [27] \\
\hline AFLA_139160 & $\operatorname{aflX}(\operatorname{ordB})$ & monooxygenase/oxidase & - \\
\hline AFLA_139170 & aflW (moxY) & monooxygenase & - \\
\hline AFLA_139180 & aflV (cypX) & cytochrome P450 monooxygenase & - \\
\hline AFLA_139200 & aflQ (ordA) & $\begin{array}{c}\text { cytochrome P450 } \\
\text { monooxygenase, CYP64 }\end{array}$ & OMST to $\mathrm{AFB}_{1}$ \\
\hline AFLA_139230 & aflI (avfA) & cytochrome P450 monooxygenase & oxidation of averufin to VHA \\
\hline AFLA_139240 & aflLa (HypB) & oxidase & oxidation of ST to AF (?) [28] \\
\hline AFLA_139250 & aflL (verB) & desaturase/P450 monooxygenase & - \\
\hline AFLA_139260 & $\operatorname{aflG}(\operatorname{avnA})$ & cytochrome P450 monooxygenase & AVN to HAVN \\
\hline AFLA_139280 & $\operatorname{aflN}(\operatorname{ver} A)$ & cytochrome P450 monooxygenase & VHA to ST \\
\hline AFLA_139400 & aflCa (HypC) & oxidase & NORA anthrone to NORA [28] \\
\hline AFLA_139430 & aflU (cypA) & cytochrome P450 monooxygenase & hydroxylate to NORA anthrone (?) \\
\hline
\end{tabular}

Moreover, nadA, a gene that encodes NADH oxidase, is located at the distal end of the aflatoxin cluster adjacent to the so-called sugar cluster. This gene encodes a protein that together with CypA catalyzes aflatoxin $\mathrm{G}$ formation [27,29]. The sugar cluster genes and nadA in particular, are co-regulated and transcribed in the same pattern as aflatoxin cluster genes [30-32]. NadA, like similar enzymes in bacteria, may contribute to generation of ROS and to alter the redox state [33]. Hence, 
a minimum of 10 enzymes associated with the aflatoxin biosynthetic pathway could potentially be a source of intracellular ROS.

\subsection{Blocking Aflatoxin Biosynthesis Limits ROS Accumulation in Whole Live Mycelia of A. parasticus}

In attempt to detect ROS in vivo, ROS staining in live $A$. parasiticus hyphae was performed using $\mathrm{H}_{2}$ DCFDA, a cell-permeable indicator for total ROS that is non-fluorescent until the acetate groups are removed by intracellular esterases and oxidation occurs within the cell. When A parasiticus SU-1 (a wild type aflatoxin producing strain), is cultured in YES (yeast extract, sucrose) liquid medium in the dark for $48 \mathrm{~h}$, it produces high levels of aflatoxin beginning at $30 \mathrm{~h}$. AFS10 (carries a deletion in aflR, that encodes a key positive regulator of aflatoxin synthesis) produces no aflatoxin while B62 generates a bright red pathway intermediate norsolorinic acid (NA) and a small quantity of aflatoxin. At $48 \mathrm{~h}$ of growth, 38-95 individual hyphae were examined in each strain after staining with $\mathrm{H}_{2} \mathrm{DCFDA}$. Approximately $87 \%$ of SU-1, 43\% of AFS10, and $48 \%$ of B 62 hyphae contained structures of the size and shape of endosomes (Figure 1A). These structures were stained green at different intensities with the highest intensity associated with endosomes in SU-1 and the weakest associated with endosomes in AFS10 (Figure 1A, arrows; Figure 1B). In B62 these endosome-like structures appear reddish-green due to accumulation of NA. In the corresponding controls (hyphae of SU-1, AFS10, and B62, with no $\mathrm{H}_{2}$ DCFDA staining) we observed little detectable fluorescence under the microscope (Nikon, Inc., Melville, NY, USA) using B2A filter set (Figure 1C). We detected only weak autofluorescence in these strains and it was not associated with endosome-like structures. Moreover, B62 demonstrated bright reddish-yellowish small patches and protrusions were located predominantly along the cell wall and detected on the outer cell surface in agreement with our previous report that aflatoxisomes carry NA to the cell exterior (Figure 1A,C) [34]. Surprisingly, these patches and protrusions were also stained with a specific fluorescent probe for autophagosomes (Cyto-ID Autophagy Detection Kit, Enzo Life Sciences, Farmingdale, NY, USA) (Figure 1Da,b), with CellROX, another marker of total ROS (Figure 1Dc), and with a lipophilic styryl dye FM4-64 (Life Technologies, Molecular Probes, Eugene, OR, USA) that stains membranes (Figure 1Dd).

A

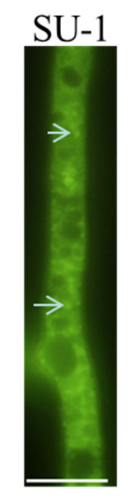

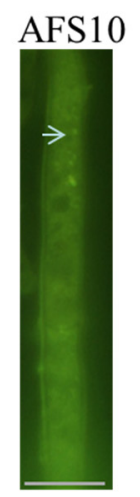

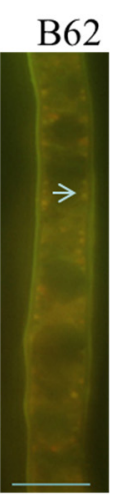

B

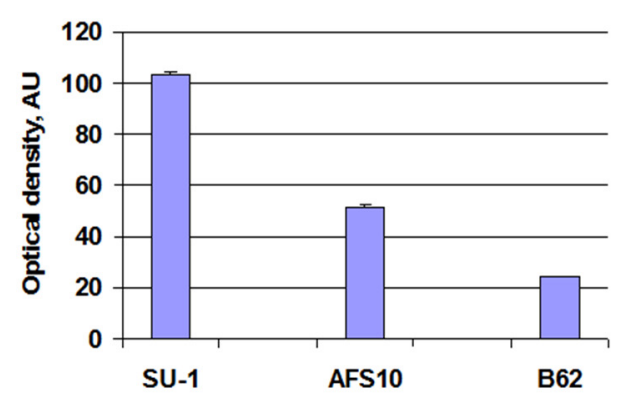

Figure 1. Cont. 


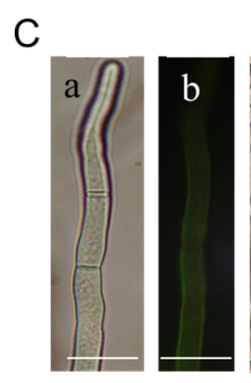

SU-1

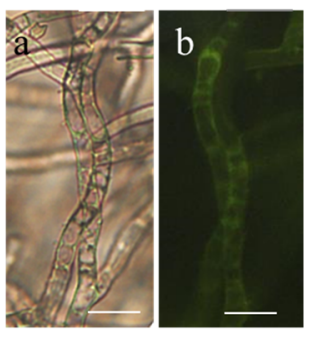

SU-1

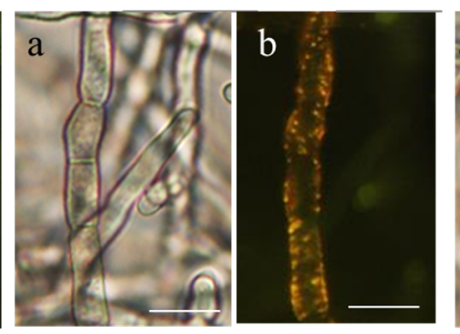

B62

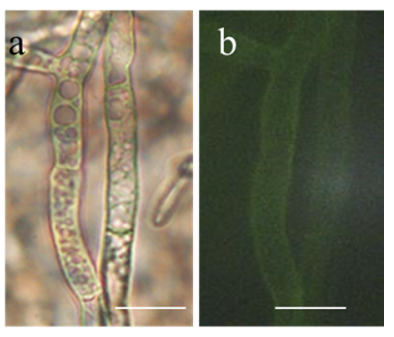

AFS10

$\mathrm{D}$
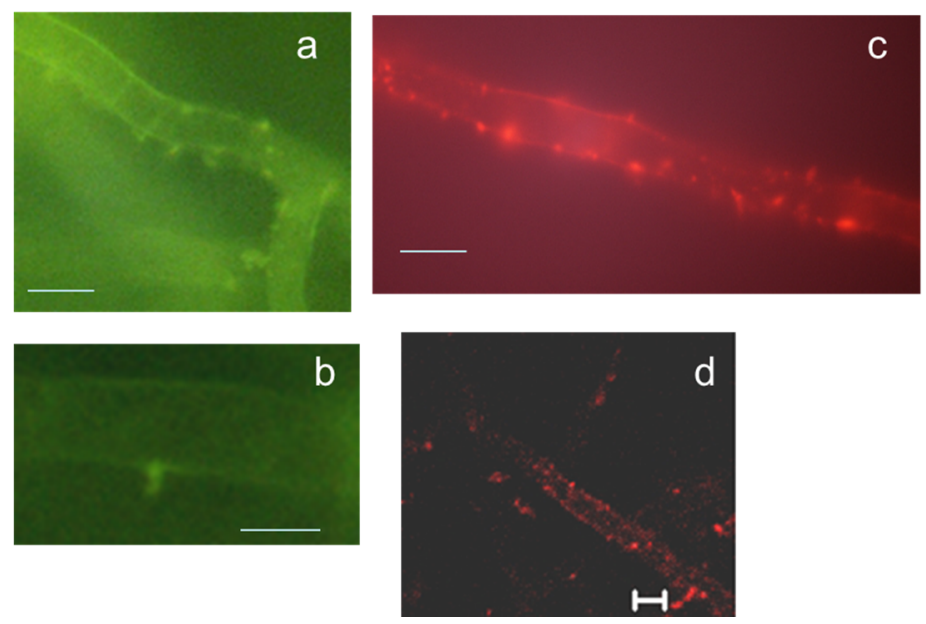

Figure 1. ROS detection using $\mathrm{H}_{2}$ DCFDA in live A. parasiticus hyphae. A. parasiticus SU-1, AFS10, and B62 spores were inoculated at $10^{4}$ spores $/ \mathrm{mL}$ into YES liquid medium and were grown at $30{ }^{\circ} \mathrm{C}, 150 \mathrm{rpm}$ in the dark for $48 \mathrm{~h}$. 6-8 colonies were incubated in the presence of $\mathrm{H}_{2}$ DCFDA $(20 \mu \mathrm{M})$ for $20 \mathrm{~min}$, washed in PBS $(0.02 \mathrm{mM}, \mathrm{pH} 7.5)$ for $5 \mathrm{~min}$ 4 times, and examined under fluorescent microscope. (A) Approximately 87\% of SU-1, 43\% of AFS 10, and 48\% of B62 hyphae contained structures of the size and shape of endosomes which stained green at different intensities with $\mathrm{H}_{2}$ DCFDA (arrows; also see (B)). In strain B62 these structures accumulate NA, the early aflatoxin pathway intermediate; (B) Quantitative imaging analysis of ROS detected by $\mathrm{H}_{2}$ DCFDA in A. parasiticus hyphae. AU, arbitrary units. Data are presented as the Mean $\pm \mathrm{SE}, N=8-12$; (C) Autofluorescence of $A$. parasiticus SU-1, AFFS10, and B62 strains used in the study observed in the absence of staining by $\mathrm{H}_{2}$ DCFDA: a, bright field image; $b$, image obtained under fluorescence microscope. Bar $100 \mu \mathrm{m}$; (D) Positive staining of protrusions with a fluorescent marker of autophagosomes and ROS. A. parasiticus SU-1 was grown for $66 \mathrm{~h}$ (as described in Figure 1) in the presence of Rapamycin ( $500 \mathrm{nM}$, added at $24 \mathrm{~h}$ to induce autophagy). 6-8 colonies were stained by Enzo Cyto-ID ${ }^{\circledR}$ Green Detection Reagent according to manufacturer's protocol. The stained cells were observed under a fluorescent microscope as described in Methods. The accumulation of the green fluorescent dye was detected in protrusions (a,b); A. parasiticus SU-1 grown for $48 \mathrm{~h}$ in liquid YES and stained with CellROX (c) or with FM4-64 (d). Bar $100 \mu \mathrm{m}$. 


\subsection{Disruption of AvaA (vb1, ypt7) Stimulates Aflatoxin Biosynthesis and ROS Accumulation in} Whole Mycelia of A. parasiticus

Disruption of avaA prevents fusion of pre-vacuolar compartments (late endosomes) to the vacuole and results in a significant increase in accumulation of endosomes and accumulation of aflatoxin in the growth medium [24]. AC11, a $\triangle a v a A(\Delta v b 1)$ strain, was grown under the same as above conditions in aflatoxin-inducing medium (liquid YES). As expected, this strain produced larger quantities of aflatoxin $\mathrm{B}_{1}$ as detected by ELISA $(2.7 \mathrm{mg} / \mathrm{mL}$ by $24 \mathrm{~h}$ of growth, and $9.3 \mathrm{mg} / \mathrm{mL}$ by $45 \mathrm{~h})$ than SU-1 $(2.49 \mu \mathrm{g} / \mathrm{mL}$ by $24 \mathrm{~h}$, and $1.27 \mathrm{mg} / \mathrm{mL}$ by $45 \mathrm{~h}$ ). At $48 \mathrm{~h}$ of growth, ROS accumulation in whole mycelia of A. parasiticus $\mathrm{AC} 11$ and $\mathrm{AC} 5$ (another $\triangle a v a A$ mutant) using $\mathrm{H}_{2} \mathrm{DCFDA}$, detected by fluorescence, was 2 to 3 fold higher than that in SU-1 (Figure 2A-C). AC11 and AC5 exhibited a characteristic "scattered bright segment" staining pattern of hyphae. Small brightly stained patches were detected in the cytoplasm and not in vacuoles in all of the strains examined. Such small brightly stained organelles exhibited the characteristic size and shape of transport vesicles and endosomes and they were found associated with the outer surface of vacuoles. AC11 and AC5 produced only weak auto-fluorescence in the absence of staining by $\mathrm{H}_{2}$ DCFDA and this was not associated with endosomes (Figure 2D).

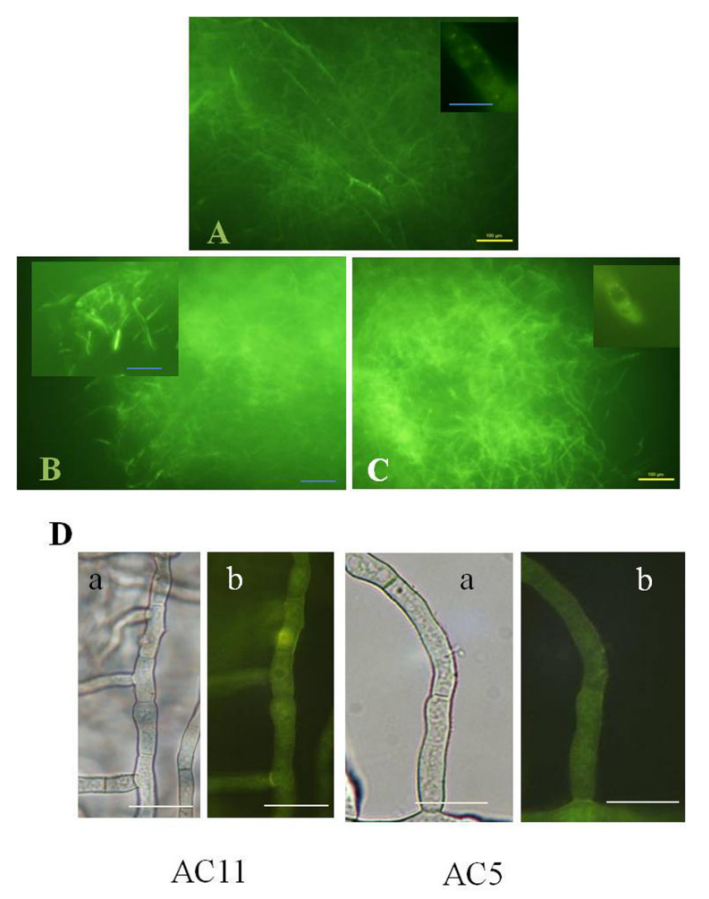

Figure 2. ROS detection using $\mathrm{H}_{2}$ DCFDA in live A. parasiticus $\mathrm{AC} 11$ and AC5 hyphae. The strains were grown and stained as described in Figure 1. Small bright dots were detected in the cytoplasm and not in vacuoles. (A) A. parasiticus SU-1; (B) A. parasiticus AC11. Characteristic "scattered bright segment" pattern of staining in cells with bright cytoplasm; the overall brightness of staining in AC11 is higher than in SU-1; (C) A. parasiticus AC5. "Scattered bright segment" staining pattern; bright green small dots surround vacuole (Inset). The overall brightness of staining in AC11 and AC5 is higher than in SU-1. Bar, $100 \mu \mathrm{m}$; (D) Autofluorescence of $A$. parasiticus AC5 and AC11 strains used in the study observed in the absence of staining by $\mathrm{H}_{2}$ DCFDA: (a), bright field image; (b), image obtained under fluorescence microscope. Bar $100 \mu \mathrm{m}$. 
D CellROX DeepRed
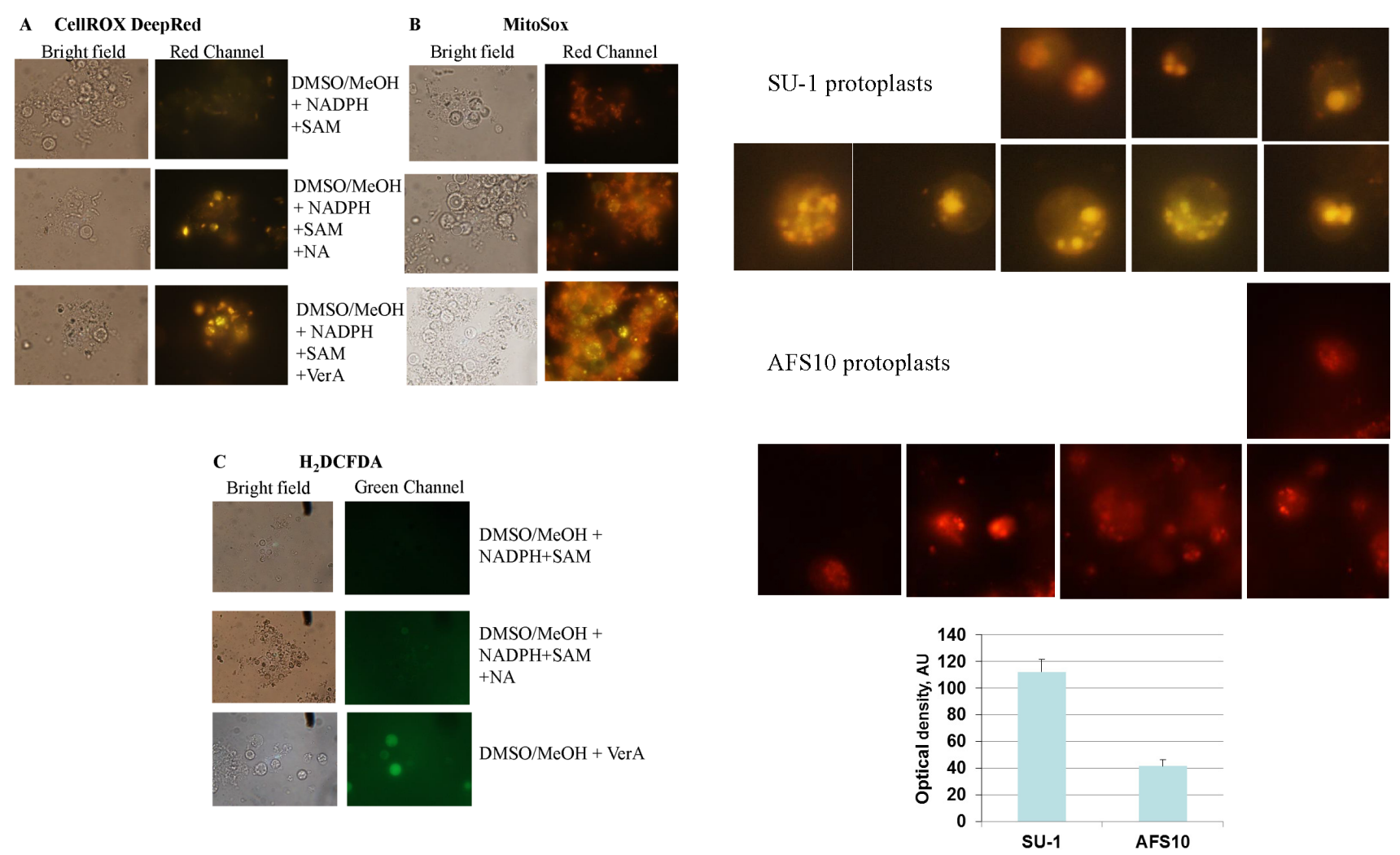

Figure 3. Feeding aflatoxin pathway intermediates to protoplasts stimulates intracellular ROS accumulation. Isolation and feeding of protoplasts were performed as described in Materials and methods. After feeding with NA and VerA the protoplasts were stained with CellROX Deep Red (A), Mitosox (B), and $\mathrm{H}_{2} \operatorname{DCFDA}(\mathbf{C})$, and visualized under a fluorescent microscope as described in Methods; (D) Comparison of the fluorescence intensity in SU-1 and AFS10 protoplasts stained with CellROX. SU-1 and AFS10 protoplasts were fed with VerA and stained using CellROX, as described in above. The images of individual protoplasts are presented in grayscale mode for assessment of the fluorescence intensity. For the graph the fluorescence intensity was assessed using ImageJ $1.48 \mathrm{v}$ software $(\mathrm{NIH}$, Bethesda, MD, USA). AU, arbitrary units. Data are presented as the Mean $\pm \mathrm{SE}, N=6$.

\subsection{Feeding Aflatoxin Pathway Intermediates to Protoplasts Stimulates Intracellular "Secondary" ROS Accumulation}

We obtained protoplasts from A. parasiticus SU-1 using standard methods [35] and fed them with the aflatoxin pathway intermediates NA (the first stable pathway intermediate) or versicolorin A (VerA, a middle pathway intermediate). Feeding with NA increased aflatoxin accumulation by 3 -fold and feeding with VerA increased it by nearly 60 -fold compared to control non-fed solvent added protoplasts. We analyzed intracellular ROS production in protoplasts using specific fluorescent dyes for superoxide (Mitosox) and for total ROS measurement ( $\mathrm{H}_{2}$ DCFDA or CellROX) after feeding for $16 \mathrm{~h}$ (Figure $3 \mathrm{~A}-\mathrm{C}$ ). Although Mitosox is used to detect superoxide in mitochondria, our data indicate that Mitosox can also be oxidized to a fluorescent product by superoxide in endosomes. Feeding with pathway intermediates increased ROS accumulation to significantly higher levels than those of control protoplasts. The ROS 
signal detected with Mitosox, CellROX and $\mathrm{H}_{2}$ DCFDA localized ROS accumulation to small organelles which exhibited the characteristic size and shape of transport vesicles and endosomes that were predominantly located near the cytoplasmic membrane. The increase in ROS was proportional to the increase in aflatoxin synthesis (i.e., greater amounts of ROS were observed with VerA than with NA) (Figure 3A-C) suggesting that enzymes involved in aflatoxin synthesis, particularly CYPs, are directly or indirectly linked to increased ROS in transport vesicles and endosomes.

Formation of ROS after addition of NA or VerA may also derive from metabolic transformation of these compounds not related to aflatoxin biosynthesis. For example, in mammalian cell culture or tissue, aflatoxin $\mathrm{B}_{1}$ can be metabolized by CYPs or lipoxygenases with formation of free radicals [36,37]. In order to distinguish ROS generated through the aflatoxin biosynthetic pathway from ROS originated in non-aflatoxin-related metabolic transformation, we fed VerA to protoplasts obtained from A. parasiticus AFS10 in which no aflatoxin biosynthetic enzymes and no aflatoxin were present. AFS10 protoplasts fed with VerA were stained with CellROX, because it generates more easily detectable fluorescence as compared with $\mathrm{H}_{2}$ DCFDA. Similar to SU-1 protoplasts, we detected ROS accumulation in AFS10 protoplasts in small organelles that exhibited a characteristic size and shape of transport vesicles and endosomes and to larger round structures; all these structures were located predominantly near the cytoplasmic membrane. However, the intensity of fluorescence (measured with Image J software, $1.48 \mathrm{v}$ ) was 2-3-fold less in AFS10 protoplasts as compared to SU-1 (Figure 3D). The data suggest that aflatoxin biosynthesis is a primary contributor to ROS accumulation in endosome-like vesicular structures and that other source of ROS exist in these organelles as well.

\subsection{Fungal Sensitivity to Hydrogen Peroxide Treatment Is Inversely Proportional to Aflatoxin Production}

We analyzed and compared the levels of tolerance to hydrogen peroxide of conidiospores and germlings of different $A$. parasiticus strains that vary in the level of aflatoxin biosynthesis. Freshly harvested conidiospores and $7 \mathrm{~h}$ germlings of $A$. parasiticus SU-1, ASF10, AC11, HypC, and $\triangle v e A$ were analyzed. These strains produce variable quantities of aflatoxin as expected depending on the mutation in the aflatoxin pathway. Of particular importance, ROS levels in these strains directly correlated with the quantities of aflatoxin produced (see above).

We also observed clear differences in catalase activity in fresh spore suspensions of $3 \mathrm{~A}$. parasiticus strains, SU-1, AFS10, and $\triangle v e A$ (Table 2). In contrast to this observation, survival of freshly harvested spores from these 3 A parasiticus strains, $\mathrm{SU}-1$, AFS10, and $\Delta v e A$, under treatment with $\mathrm{H}_{2} \mathrm{O}_{2}$ was not significantly different (data not shown) suggesting that hydrogen peroxide may not enter freshly harvested spores. In support of this idea, when we germinated freshly harvested spores of five different A. parasiticus strains for $7 \mathrm{~h}$ in five independent experiments, we observed unique and reproducible trends in response to oxidative stress. AC11 which produces the largest quantities of aflatoxin had the highest tolerance to all concentrations of hydrogen peroxide tested and $\Delta v e A$ in which no aflatoxin is produced was the most sensitive to hydrogen peroxide treatment (Figure 4a-e). Overall, we observed a strong inverse correlation between the quantity of aflatoxin produced by the strain and the sensitivity to hydrogen peroxide. The largest differences in sensitivity to hydrogen peroxide treatment were observed at the highest concentration of hydrogen peroxide tested, $100 \mathrm{mM}$ (Figure 4f), and SU-1 (produced 
$2.49 \mu \mathrm{g} / \mathrm{mL}$ of aflatoxin $\mathrm{B}_{1}$ by $24 \mathrm{~h}$, and $1.27 \mathrm{mg} / \mathrm{mL}$ of aflatoxin $\mathrm{B}_{1}$ by $45 \mathrm{~h}$ ) survived at 2-fold or higher levels than AFS10 or $\triangle v e A$.
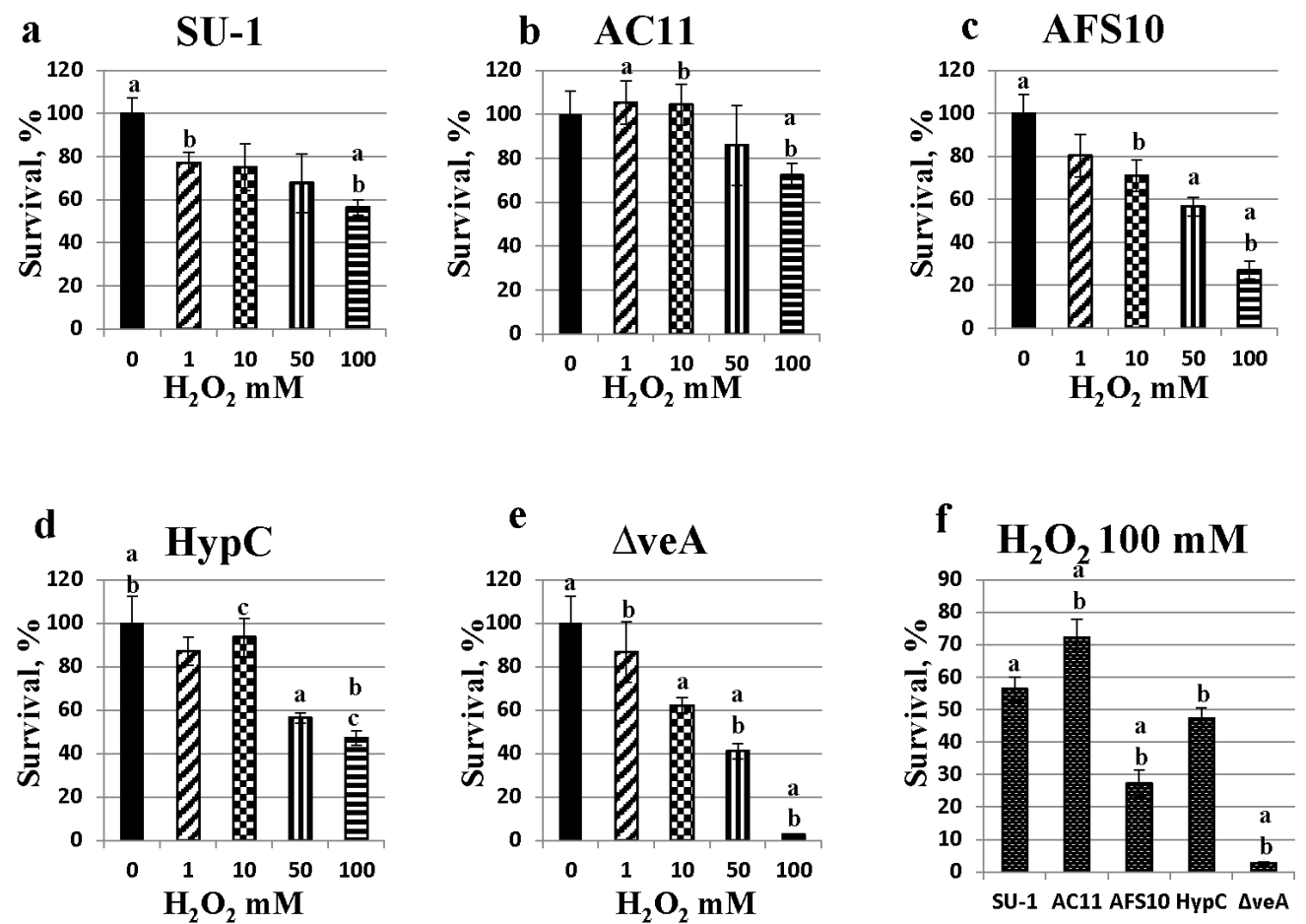

Figure 4. Tolerance of 7-h germlings to treatment with $\mathrm{H}_{2} \mathrm{O}_{2}$. A . parasiticus $\mathrm{SU}-1$, AFS10, $\mathrm{AC} 11, \mathrm{HypC}$, and $\triangle v e A$ conidiospores (grown and freshly harvested as described in Methods) were incubated for $7 \mathrm{~h}$ in GMS liquid medium and then exposed to $1,10,50$, or $100 \mathrm{mM}$ hydrogen peroxide for $30 \mathrm{~min}$. Viability of germlings was analyzed by growth of treated and untreated (control) germlings on YES agar growth medium which was incubated at $30{ }^{\circ} \mathrm{C}$ for $72 \mathrm{~h}$. Fungal colonies were counted and \% survival was calculated as follows: cfu treated/ cfu untreated $\times 100$. Experiment was performed 5 times using 3 replicates with similar trends. Results of a representative experiment are presented as Mean \pm SE. An identical letter above the bar shows statistically significant difference in survival between the indicated data points $(p<0.05)$; statistical analysis was performed by pairwise Student's t-test (SigmaStat) and by two-way ANOVA and Tukey's HSD test (R Statistical Software, R-3.1.1).

Table 2. Catalase activity in fresh conidiospores. Catalase activity was determined in a suspension of freshly harvested conidiospores by an assay provided in Worthington Enzyme Manual as described in Methods. ). The experiment was performed 3-4 times. The activity was expressed in micromoles $\mathrm{H}_{2} \mathrm{O}_{2}$ degraded per min per $10^{4}$ spores at $25^{\circ} \mathrm{C}$ and $\mathrm{pH} 7.0$, and presented as Mean \pm SE. ND not detected. *, statistically significant difference as compared to SU-1, $p=0.0002$. Statistical analysis was performed by Student's $t$-test using SigmaStat 1.0).

\begin{tabular}{ccc}
\hline SU-1 & AFS10 & $\Delta v e \boldsymbol{A}$ \\
\hline $3.0 \pm 0.1$ & $* 1.6 \pm 0.1$ & ND \\
\hline
\end{tabular}


Table 3. Primer sequences used for transcript analysis.

\begin{tabular}{cccc}
\hline Gene & Primer sequence $^{\text {a }}$ & PCR product $^{\mathbf{b}}$ (bp) & Intron $^{\mathbf{c}}$ \\
\hline Citrate Synthase & F-5' TGCAGTCCGTTGCCTTCAATG 3' & \multirow{2}{*}{$518(827)$} & 5 \\
AFLA_007020 & R-5' TAGCGTAGGCCTTGGCGAAAG 3' & & \\
Cat Spore & F-5' AATGTACAGTCCAGCAAGG 3' & $537(648)$ & 2 \\
AFLA056170 & R-5' CGGGTGAAGATAGACAAAG 3' & & \\
Cat Mycelia & F-5' ATGACACATTCCTGACCTC 3' & \multirow{2}{*}{$456(583)$} & 2 \\
AFLA090690 & R-5' TCTCATTGTCACCATGAG 3' & & \multirow{2}{*}{2} \\
SOD Mn & F-5' CATTCTCCCTCCCACCTCTC 3 & $549(676)$ \\
AFLA033420 & R-5'TCCAGATGCCCTCCACATAC 3' & & \\
SOD Fe & F-5' TGGGAGAGTTCCAGAGCAAG 3' & $530(796)$ & 3 \\
AFLA027580 & R-5' TGTCGATGCCTTTCGGAG 3' & & \\
SOD Cu/Zn cytosol & F-5' GAAGCTGTTCTCCAGGAC 3' & $434(492)$ & 1 \\
AFLA068080 & R-5' GACACCAGGTGGAAGTTAC 3' & \\
\hline
\end{tabular}

${ }^{\mathbf{a}} \mathrm{F}$ represents forward primers and R represents reverse primers; ${ }^{\mathbf{b}}$ The size of PCR products obtained using genomic DNA (gDNA) as template is depicted in parenthesi; ${ }^{\mathbf{c}}$ The number of introns spanned by primers.

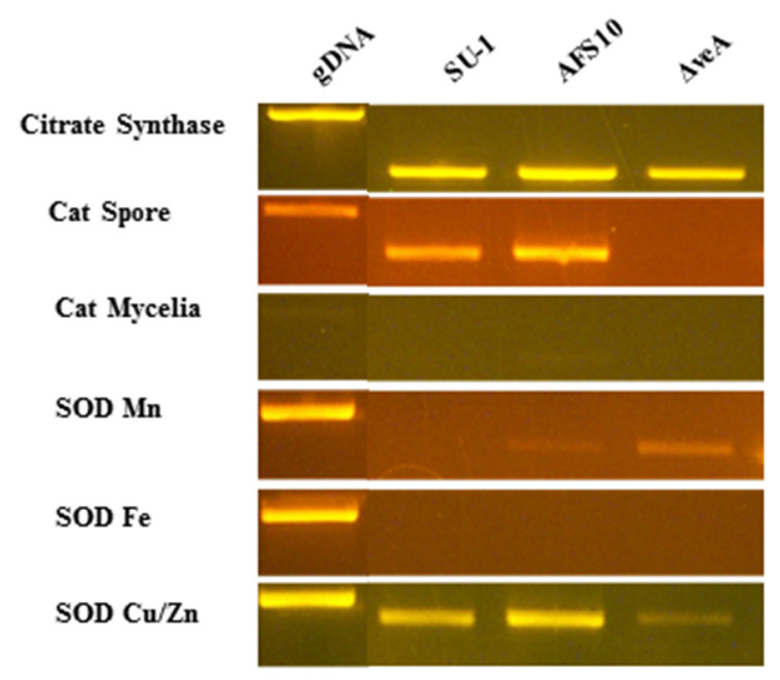

Figure 5. Analysis of transcript levels in $7 \mathrm{~h}$ germlings. A. parasiticus SU-1, AFS10, and $\Delta v e A$ were grown on YES solid media and spores were collected at 5 days. Germlings were grown from fresh spores in GMS liquid medium for $7 \mathrm{~h}$, frozen in liquid nitrogen, and stored at $-80^{\circ} \mathrm{C}$. RNA was extracted from germlings using grinding and sonication as described in Methods. RT-PCR was performed on total RNA treated with RNAse-free DNAse I with primers specific for each gene (Table 3). PCR products were separated on a 1\% agarose gel by electrophoresis. Citrate synthase, a constitutively expressed gene, was used as a positive control. gDNA, genomic DNA.

\subsection{Transcript Accumulation in Seven-Hour Germlings}

We measured total catalase activity in freshly harvested conidiospores in SU-1, AFS10, and $\Delta v e A$, and observed a direct correlation between catalase activity and resistance to hydrogen peroxide in $7 \mathrm{~h}$ germlings. However, we were unable to isolate RNA from conidiospores. To determine if this difference in catalase activity is regulated at the level of transcript accumulation, we analyzed expression of 
3 catalases, catalase spore (AFLA_056170), catalase putative (AFLA_034380), catalase mycelial (AFLA_090690), and 3 superoxide dismutases, SODs, including SOD Mn (AFLA_033420), SOD Fe (AFLA_027580), and SOD Cu/Zn (AFLA_068080) in $7 \mathrm{~h}$ germlings of SU-1, AFS10, and $\Delta v e A$ using semi-quantitative PCR. Catalase putative transcript was detected in A. flavus $(68 \mathrm{~h})$ but not in A. parasiticus strains studied (not shown). Disruption of $v e A$ caused a significant decrease in transcript accumulation of spore catalase and $\mathrm{Cu} / \mathrm{Zn}$ SOD in $7 \mathrm{~h}$ germlings as compared to SU-1 and AFS10 (Figure 5). However, transcript accumulation of SOD $\mathrm{Mn}$ in $\Delta v e A$ increased. Unfortunately, the expression data did not provide additional clues about the mechanisms of resistance to hydrogen peroxide in $7 \mathrm{~h}$ germlings.

\section{Discussion}

Increasing experimental evidence establishes that secondary metabolism contributes to the long term survival of the producing organism despite the fact that it can grow and reproduce in the absence of synthesis of secondary metabolites [38-40]. Aflatoxin biosynthesis requires a significant investment of energy, building materials, and the use of cellular organelles, in order to be completed. All these suggest that aflatoxins could be of biological importance to the producing fungus.

Our data provide support to the hypothesis that the aflatoxin biosynthetic pathway generates a redox signal in endosomes and this enhances resistance to oxidative stress in germinating conidiospores. We utilized mutants that produce different levels of aflatoxin, as well as specific dyes, Mitosox in particular, to provide data that suggest that the predominant ROS generated by the aflatoxin pathway is superoxide. Since superoxide radical is readily converted by SOD to hydrogen peroxide, the presence of hydrogen peroxide is also anticipated. Feeding experiments provided supporting evidence that the aflatoxin biosynthetic pathway generates ROS, presumably superoxide and hydrogen peroxide.

Secondary metabolism in A. parasiticus SU-1 generates multiple toxic and/or mutagenic compounds including aflatoxin, sterigmatocystin, and, as our data suggest, ROS. In order to protect itself from the damaging effects of these toxic chemicals, the fungus uses available cellular machinery such as compartmentalization, exocytosis, and antioxidant enzymes to manage exposure of the fungal cell to these compounds. Previous studies demonstrate that aflatoxin biosynthesis is carried out in specific sub-cellular compartments called aflatoxisomes and that these compartments also participate in storage and export of aflatoxin and other substances, such as aflatoxin enzymes, to the cell exterior [34]. The filamentous fungus Fusarium graminearum, a causative agent of head blight in wheat, apparently uses very similar compartments to fulfill biosynthesis of trichothecenes; these compartments have been named "toxisomes" and were shown to differ from peroxisomes [41]. Of particular importance, two enzymes involved in trichothecene biosynthesis, Tri4p and Tri1p, which are members of a P450 superfamily, co-localize in toxisomes. Future studies will determine if Fusarium, like Aspergillus, is capable of generating a ROS redox signal in toxisomes.

In the current study, vesicles that protrude through the cell surface outward were positively stained with CellROX and by a specific fluorescent marker of autophagosomes .These data suggest that autophagy may play a role in aflatoxin biosynthesis and autophagosomes may represent one sub-fraction of heterogeneous population of aflatoxisomes that participate in exocytosis of ROS and aflatoxins thus 
supporting the hypothesis. Antioxidant enzymes, catalase, and SOD, detected in endosomes/aflatoxisomes [42], may provide an additional control to the levels of ROS in endosomes.

Several previous studies suggested that aflatoxin biosynthesis participates in signaling functions. For example, aflatoxin biosynthesis intermediates were linked to sclerotia development; however, the mechanism of this regulation was not reported [25,43]. Similarly, conidiospore development and aflatoxin biosynthesis have been shown to be co-regulated [44]. Furthermore, biosynthesis of aflatoxin was not the only secondary metabolic pathway linked to development. Forseth and co-authors showed that two secondary metabolite clusters, $\ln a$ and $\ln b$, located on different chromosomes and containing two non-canonical non-ribosomal peptide synthetase (NRPS) genes, participate in regulation of sclerotia production in Aspergillus flavus [45]. Moreover, adducts of the secondary metabolites diorcinol and dehydroaustinol triggered initiation of sporulation in $A$. nidulans [46]. In addition, sclerotia development in A. flavus is responsive to ROS, in particular to hydrogen peroxide [10]. Our studies may provide a possible mechanistic link between the regulatory circuits for aflatoxin biosynthesis and development; this link is most likely secondary ROS generated by the biosynthetic pathway with the involvement of P450s.

It is clear that aflatoxin biosynthesis is regulated by oxidative stress (30-36). Recent studies by Grintzalis and co-workers demonstrated that a group of ROS including superoxide is involved in that regulation [10]. We speculate that secondary ROS may provide a feedback mechanism to self-activate the biosynthetic pathway.

Our data demonstrate a strong association between aflatoxin synthesis and resistance to oxidative stress (hydrogen peroxide) in germlings. The transcripts and the proteins encoded by aflatoxin biosynthetic genes are not detected in 7-h germlings but begin to accumulate in the fungal mycelium later, between $24 \mathrm{~h}$ and $30 \mathrm{~h}$ of growth [47]. The accumulation of transcripts and proteins correlates with accumulation of aflatoxin. For this reason, we suggest that pre-exposure of fungal cells to ROS generated in endosomes during aflatoxin biosynthesis in parental cells results in adaptation of cellular metabolism to promote increased tolerance to $\mathrm{H}_{2} \mathrm{O}_{2}$ in the next generation (young germlings). Therefore, our work may illustrate a novel function for aflatoxin biosynthesis. Specifically, the data suggest that aflatoxin biosynthesis provides protection and a competing advantage. In support of this idea, development of adaptive tolerance to stress by exposure to low levels of hydrogen peroxide was demonstrated previously in fibroblasts, bacteria, and plants [48-51].

To summarize progress in our understanding of the role of ROS in fungi, we provide a regulatory model that emphasizes a key role for endosomes and associated secondary ROS in redox signaling (Figure 6). According to this model, extracellular ROS influence at least two signaling pathways. First, a NoxA initiated burst of ROS that we designate as "primary ROS burst", modulates activity of AflR, a pathway-specific transcription factor that regulates aflatoxin biosynthesis. The SAPK/MAPK pathway, the second signaling pathway, activates the bZIP transcription factor AtfB, and promotes complex formation between AflR and AtfB at promoters of genes involved in aflatoxin synthesis, in promoters of antioxidant genes such as catalase and SOD, and promoters of genes involved in sclerotia/conidiospore development. Antioxidant and aflatoxin biosynthetic enzymes localize to transport vesicles which fuse to form endosomes. Activities of CYPs for aflatoxin biosynthesis, SOD, and catalase in endosomes generate and modulate a "secondary ROS burst" and subsequently a redox signaling from endosomes mediates development related processes (sclerotia and conidiospore development). The secondary ROS burst transmits increased tolerance to oxidative stress to the next generation of germlings. Endosomes eventually fuse with the cytoplasmic membrane to export their contents by exocytosis. Limitation for 
carbon stimulates aflatoxin synthesis and may also stimulate autophagy as aflatoxin synthesis is winding down when endosomes fuse with vacuoles.

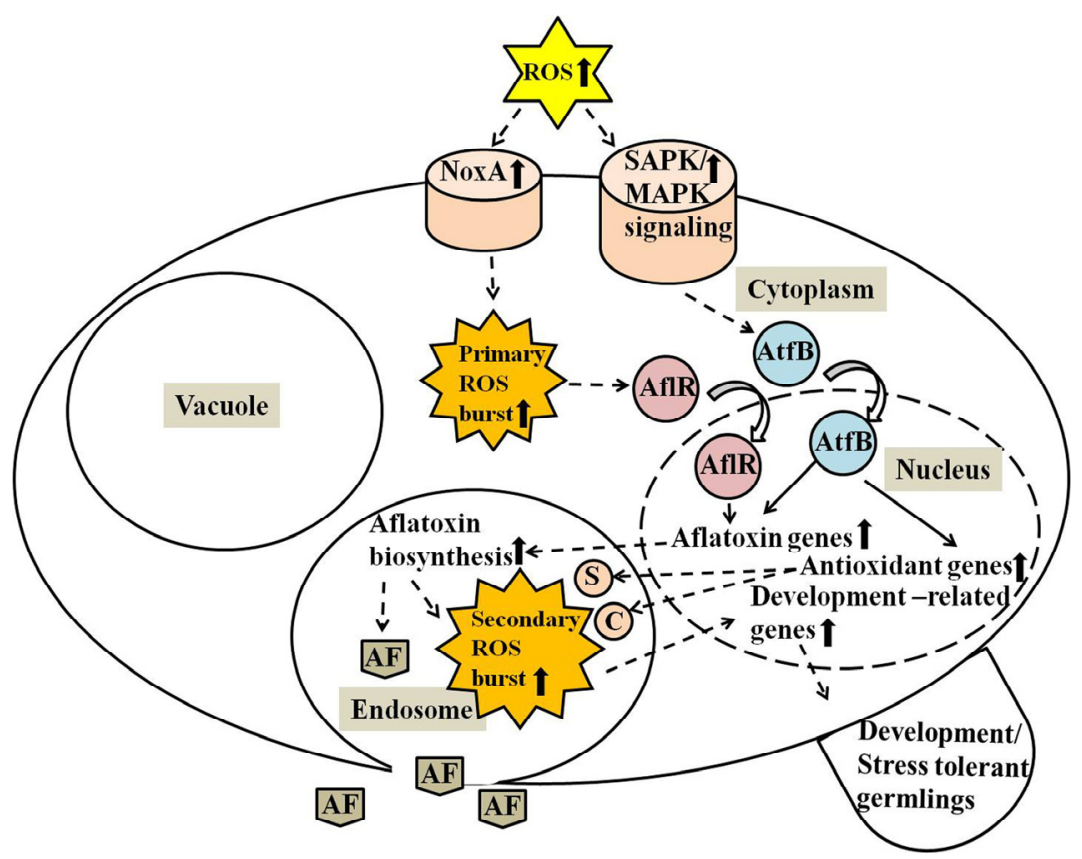

Figure 6. Secondary ROS and signaling from endosomes in aspergilli. Proposed model for stress-tolerant fungal germling development by ROS generated during secondary metabolism is based on available experimental data including current studies. The model proposes that increased levels of extracellular ROS up-regulate NADPH oxidase A (NoxA) and the stress activated protein kinase/mitogen activated protein kinase (SAPK/MAPK) signaling pathway. Activation of the SAPK/MAPK signaling cascade promotes AtfB binding to corresponding CRE sites in promoters of antioxidant and aflatoxin genes. NoxA induces intracellular primary ROS bursts that in turn activate AflR, which binds to corresponding AFLR sites in promoters of aflatoxin biosynthetic genes for activation of the gene expression. Aflatoxin biosynthetic enzymes are transported into endosomes, where aflatoxins (AF) are produced, and then secreted into medium concurrently with the toxin. Secondary ROS bursts produced during aflatoxin production in endosomes/aflatoxisomes, by most likely epigenetic mechanisms convey $\mathrm{H}_{2} \mathrm{O}_{2}$ resistance to next generation germlings. The dotted lined arrows indicate signal transduction and the solid lined arrows indicate binding of transcription factors, AtfB and AflR, to the corresponding sites in the promoters of the genes. The curved arrows designate entering of the transcription factors from cytoplasm to nucleus. Superoxide dismutase $(\mathrm{S})$ and catalase $(\mathrm{C})$ present in aflatoxisomes control levels of secondary ROS and associated signaling.

Thus, secondary metabolism and secondary ROS in particular, may have the potential to regulate environmental fitness of the cell. Our work reveals the idea that aflatoxin biosynthesis-related secondary ROS may contribute to signaling in long term cell survival. Therefore, protection of cells from chemically unstable ROS (which makes the toxin) may have detrimental consequences to the survival of the cell. 


\section{Materials and Methods}

\subsection{Strains, Media, Growth Conditions}

The $A$. parasiticus strains SU-1 (a wild type aflatoxin producing strain), A. parasiticus AFS10 (deletion in aflR, a key positive regulator of aflatoxin synthesis, produces no aflatoxin), A. parasiticus B62 (point mutation in nor-1, accumulates the bright red pathway intermediate norsolorinic acid (NA) and a small quantity of aflatoxin), A. parasiticus AC11 [24] (disruption of avaA, produces higher quantities of aflatoxin and increased toxin export of aflatoxin compared to SU-1), A. parasiticus $\Delta$ hypC [28] (accumulates smaller quantities of aflatoxin than wild type), and $A$. parasiticus $\Delta v e A$ [52] (produces no secondary metabolites including aflatoxin) were used. YES, a rich medium, and GMS, a chemically defined medium, were used as aflatoxin-inducing media.

\subsection{Detection of ROS in Live Cells}

$\mathrm{H}_{2}$ DCFDA, CellROX DeepRed and Mitosox were purchased from Invitrogen (Molecular Probes, Eugene, OR, USA). Whole mycelia and protoplasts were incubated with dyes in the growth medium for 10-15 min and washed in the medium 3 times according to the manufacturer's instructions. Samples were observed and photographed under a Nikon fluorescence microscope using a filter with $495 \mathrm{~nm}$ excitation and 500-550 nm emission wavelengths ( $\mathrm{H}_{2}$ DCFDA and Mitosox staining) or a filter with $510 \mathrm{~nm}$ excitation and $580 \mathrm{~nm}$ emission wavelengths (CellROX Deep Red staining).

\subsection{Measuring Catalase and SOD Activities}

Activities of catalase and superoxide dismutase (SOD) were measured by protocols described in Worthington Enzyme Manual available online (http://www.worthington-biochem.com/CTL/assay.html). Catalase activity assay. The catalase assay is based on measuring the decrease of hydrogen peroxide levels during a reaction by means of a spectrophotometer $(240 \mathrm{~nm})$. The activity was expressed in $\mu \mathrm{mol}$ $\mathrm{H}_{2} \mathrm{O}_{2}$ degraded per min per milligram of total protein at $25^{\circ} \mathrm{C}$ and $\mathrm{pH}$ 7.0.

To measure catalase activity using a crude protein extract from fungal mycelium, $A$. parasiticus spores were inoculated at $10^{5}$ spores/mL and grown in YES liquid medium for designated time in the dark with shaking at $150 \mathrm{rpm}$. The mycelium was harvested through Miracloth (Calbiochem, EMD Chemicals, San Diego, CA, USA) and frozen in liquid $\mathrm{N}_{2}$. Frozen mycelium was ground in liquid $\mathrm{N}_{2}$ in a mortar with a pestle and the powdered mycelium was resuspended $1: 1(w / v)$ in TSA buffer $(0.01 \mathrm{M}$ Tris, $0.15 \mathrm{M} \mathrm{NaCl}, 0.05 \% \mathrm{NaN}_{3}, \mathrm{pH} 8.0$ ) containing 1 tablet of Complete Mini Protease Inhibitor cocktail (Roche Diagnostics, Mannheim, Germany), $50 \mu$ L Proteinase Inhibitors mix (Sigma, St. Louis, MO, USA), and $0.125 \mathrm{mM}$ phenylmethylsulphonyl fluoride (Sigma, St. Louis, MO, USA) per $10 \mathrm{~mL}$. The cell lysate was centrifuged at $13,000 \mathrm{rpm}$ at $+4{ }^{\circ} \mathrm{C}$ for $15 \mathrm{~min} .50 \mu \mathrm{L}$ of $1 / 10$ diluted supernatant was added to a mix containing $0.95 \mathrm{~mL}$ of distilled sterile water and $0.5 \mathrm{~mL}$ of $0.059 \mathrm{M}$ hydrogen peroxide in $0.05 \mathrm{M}$ potassium phosphate (Mallinkrodt Baker, Phillipsburg, NJ, USA) pH 7.0. Decrease in absorbance at $240 \mathrm{~nm}$ was recorded using spectrophotometer Spectronic 601 (Milton Roy, Ivyland, PA, USA). 
To assess catalase activity in conidiospores, freshly harvested spores (fungus was grown on GMS or YES agar medium at $30^{\circ} \mathrm{C}$, in dark (SU-1, AFS10), or light ( $\left.\Delta v e A\right)$ for 7 days) were kept frozen at $-80{ }^{\circ} \mathrm{C}$ before the assay as described above; $10^{6}$ or $10^{7}$ spores in $50 \mu \mathrm{L}$ were used per reaction mix. The activity is expressed in micromoles $\mathrm{H}_{2} \mathrm{O}_{2} / \mathrm{min} / 10^{4}$ spores.

Superoxide dismutase activity assay. The superoxide dismutase assay is based on measuring the inhibition rate of the production of blue formazan from nitro-blue tetrazolium reduction in the presence of superoxide. The maximum production of formazan was observed in the absence of the enzyme. The enzyme activity was expressed in Units per milligram of total protein, where one Unit is defined as enzyme quantity that is capable to inhibit nitro-blue tetrazolium reduction by $50 \%$. Briefly, for each reaction mix, the following reagents were combined in a glass test tube: $0.1 \mathrm{~mL}$ of $0.1 \mathrm{M}$ EDTA containing $0.3 \mathrm{mM} \mathrm{NaCN}$ (Mallinkrodt Baker, Phillipsburg, NJ, USA), $50 \mu \mathrm{L}$ of $1.5 \mathrm{mM}$ nitroblue tetrazolium (Sigma-Aldrich, St. Louis, MO, USA), $30 \mu \mathrm{L}$ of cell lysate or spore suspension (or water in control), and $1.245 \mathrm{~mL}$ of $0.067 \mathrm{M}$ potassium phosphate buffer, $\mathrm{pH}$ 7.8. The reaction was initiated by adding $75 \mu \mathrm{L}$ of $0.12 \mathrm{mM}$ riboflavin (Sigma-Aldrich, St. Louis, MO, USA) at timed intervals. The tubes were incubated on light for $12 \mathrm{~min}$ and the A560 was recorded every minute over 12 min interval.

\subsection{Analysis of Aflatoxin Accumulation}

Quantity of aflatoxin $\mathrm{B}_{1}$ in the growth medium was estimated using ELISA as described previously [30].

\subsection{Analysis of Transcript Accumulation}

At designated time points spores, germlings, or mycelia were frozen in liquid nitrogen and total RNA was extracted immediately from frozen cells using the Trizol reagent (Life Technologies, Carlsbad, GA, USA) and a protocol described previously [30]. RT-PCR was performed on total RNA treated with RNAse-free DNAse I (Roche Diagnostics, Indianapolis, IN, USA) with primers specific for the coding region of each gene. PCR products were separated on a $1 \%$ agarose gel by electrophoresis. The primer sequences used for transcript analyses are shown in Table 3.

\subsection{Feeding Protoplasts with Aflatoxin Biosynthesis Intermediates}

Protoplasts isolation and feeding with norsolorinic acid (NA) and versicolorin A (VerA) were performed as described previously [42]. Briefly, the protoplasts suspension $\left(10^{6} / \mathrm{mL}\right)$ was aliquoted by $50 \mu \mathrm{L}$. The following compounds (as indicated) were added to the protoplasts: Control, $10 \mu \mathrm{L}$ of DMSO/MeOH (1:1); $10 \mu \mathrm{L}$ of $1 \mathrm{mM}$ NADPH stock; $1 \mu \mathrm{L}$ of $32 \mathrm{mM}$ S-adenosylmethionine stock [53]; $40 \mu \mathrm{g}$ of NA in $10 \mu \mathrm{L}$ DMSO/MeOH; $243 \mu \mathrm{g}$ of versicolorin A (VerA) in $10 \mu \mathrm{L}$ DMSO/MeOH. NADPH and SAM are cofactors required for activity of enzymes in aflatoxin biosynthetic pathway [54,55]. The tubes with protoplasts were incubated at $30^{\circ} \mathrm{C}$ for $1 \mathrm{~h}$. All chemicals were from Sigma-Aldrich.

\subsection{Assessment of Fungal Resistance to Hydrogen Peroxide Treatment}

The first protocol describes treatment of conidiospores by hydrogen peroxide, while the second protocol was used to perform treatment of $7 \mathrm{~h}$ germlings. Protocol 1 . A. parasiticus conidiospores $\left(5 \times 10^{4}\right.$ spores in $\left.50 \mu \mathrm{L}\right)$ were spread onto YES agar medium and grown for 5 days at $30{ }^{\circ} \mathrm{C}$; 
A. parasiticus SU-1 was grown in the dark while $A$. parasiticus $\Delta v e A$ was grown in the light. Fresh spores $\left(10^{6} / \mathrm{mL}\right)$ were harvested and treated in the presence of various concentrations of $\mathrm{H}_{2} \mathrm{O}_{2}$ for $30 \mathrm{~min}$ at $30^{\circ} \mathrm{C}$, washed two times with water, spread onto GMS agar plates, and the number of colonies was counted after $16 \mathrm{~h}$ of incubation at $30{ }^{\circ} \mathrm{C}$ in the dark. Protocol 2. A. parasiticus conidiospores $\left(5 \times 10^{4}\right.$ spores in $\left.50 \mu \mathrm{L}\right)$ were spread onto YES agar medium and grown for 5 days at $30{ }^{\circ} \mathrm{C}$; A. parasiticus SU-1 was grown in the dark while $A$. parasiticus $\Delta v e A$ was grown in the light. Freshly harvested A. parasiticus conidiospores were inoculated into GMS liquid medium $\left(10^{4} / \mathrm{mL}\right.$ final concentration) and grown for $7 \mathrm{~h}$ at $30^{\circ} \mathrm{C}$ with shaking at $150 \mathrm{rpm}$ in the dark. Seven-hour germlings were incubated in the presence of various concentrations of $\mathrm{H}_{2} \mathrm{O}_{2}$ for $30 \mathrm{~min}$ at $30^{\circ} \mathrm{C}$ in the dark, washed two times with water, spread onto GMS agar plates, and the number of colonies was counted after $16 \mathrm{~h}$ of incubation at $30^{\circ} \mathrm{C}$ in the dark. A. parasiticus $\Delta v e A$ was grown in the light.

\subsection{Microscopy}

For fluorescence microscopy, the samples were observed under a Nikon Labophot fluorescence microscope (Nikon Inc., Melville, NY, USA) using B2A filter set (EX 450-490 nm, EM above 520). The images were acquired with the Nikon Digital Sight Camera System (Nikon Inc., Melville, NY, USA). Quantitative measurements of the fluorescent signal of intracellular endosome-like structures were performed using grayscale mode and Adobe Photoshop 6.0 software (Adobe Systems Inc., San Jose, CA, USA). Mean values of 8-12 individual measurements were averaged and background (area containing no cells) was subtracted. Data are presented as Mean $\pm \mathrm{SE}$.

\subsection{Statistical Analysis}

Statistical analyses were performed by Student's $t$-test using SigmaStat Scientific statistical software (version 1.0, Jandel Corporation, San Rafael, CA, USA) and by two-way ANOVA and Tukey's HSD test (R Statistical Software, R-3.1.1, R Foundation for Statistical Computing, Vienna, Austria, 2013).

\section{Conclusions and Perspectives for Future Studies}

Our studies provide two main conclusions. (1) The aflatoxin biosynthetic pathway in A parasiticus directly or indirectly generates ROS in aflatoxisomes/endosomes; and (2) Superoxide represents a significant fraction of the aflatoxin biosynthesis-derived ROS pool.

The ability of the aflatoxin biosynthetic pathway to generate ROS in endosomes with signaling function opens up a new direction for work on the secondary metabolism and its significance for development and cell survival. Although several fungal species are able to synthesize aflatoxins and structurally related compounds [47], fungal genomes include a large number of different secondary metabolic pathways in addition to the aflatoxin cluster [44,56]. Moreover, biosynthesis of secondary metabolites comprises various oxidative reactions with the participation of cytochrome P450s [57]. Most likely, the aflatoxin biosynthetic pathway is unique in the sense that it carries the highest number of cytochrome P450s among secondary metabolic pathways. Out of a total of approximately 150 ORFs identified as cytochrome P450s in the A. flavus genome, seven of these genes are present in the aflatoxin gene cluster (http://www.aspergillusflavus.org/genomics/). Hence, secondary metabolic pathways may 
provide continuous input to the intracellular pool of ROS and associated redox signaling. Future studies will attempt to uncover the specific mechanisms underlying secondary ROS signaling and its significance.

\section{Acknowledgments}

This work was supported by NIH grant CA 52003-21 (J.E.L.) and Michigan State University AgBio Research.

\section{Author Contributions}

L.V.R. and J.E.L conceived and designed the experiments; L.V.R., M.L., S-Y.S, J.W, S-S.W, B.V., D.A. performed the experiments; L.V.R., K.C.E., and J.E.L. analyzed the data; K.C.E. contributed reagents/materials/analysis tools; L.V.R. and J.E.L. wrote the paper.

\section{Conflicts of Interest}

The authors declare no conflict of interest.

\section{References}

1. Malagnac, F.; Lalucque, H.; Lepere, G.; Silar, P. Two NADPH oxidase isoforms are required for sexual reproduction and ascospore germination in the filamentous fungus Podospora anserina. Fungal Genet. Biol. 2004, 41, 982-997.

2. Aguirre, J.; Lambeth, J.D. Nox enzymes from fungus to fly to fish and what they tell us about Nox function in mammals. Free Radic. Biol. Med. 2010, 49, 1342-1353.

3. Lara-Ortiz, T.; Riveros-Rosas, H.; Aguirre, J. Reactive oxygen species generated by microbial NADPH oxidase NoxA regulate sexual development in Aspergillus nidulans. Mol. Microbiol. 2003, 50, 1241-1255.

4. Cano-Dominguez, N.; Alvarez-Delfin, K.; Hansberg, W.; Aguirre, J. NADPH oxidases NOX-1 and NOX-2 require the regulatory subunit NOR-1 to control cell differentiation and growth in Neurospora crassa. Eukaryot. Cell 2008, 7, 1352-1361.

5. Osiewacz, H.D.; Scheckhuber, C.Q. Impact of ROS on ageing of two fungal model systems: Saccharomyces cerevisiae and Podospora anserina. Free Radic. Res. 2006, 40, 1350-1358.

6. Giesbert, S.; Schurg, T.; Scheele, S.; Tudzynski, P. The NADPH oxidase Cpnox1 is required for full pathogenicity of the ergot fungus Claviceps purpurea. Mol. Plant Pathol. 2008, 9, 317-327.

7. Egan, M.J.; Wang, Z.Y.; Jones, M.A.; Smirnoff, N.; Talbot, N.J. Generation of reactive oxygen species by fungal NADPH oxidases is required for rice blast disease. Proc. Natl. Acad. Sci. USA 2007, 104, 11772-11777.

8. Kim, H.J.; Chen, C.; Kabbage, M.; Dickman, M.B. Identification and characterization of Sclerotinia sclerotiorum NADPH oxidases. Appl. Environ. Microbiol. 2011, 77, 7721-7729.

9. Kayano, Y.; Tanaka, A.; Akano, F.; Scott, B.; Takemoto, D. Differential roles of NADPH oxidases and associated regulators in polarized growth, conidiation and hyphal fusion in the symbiotic fungus Epichloe festucae. Fungal Genet. Biol. 2013, 56, 87-97. 
10. Grintzalis, K.; Vernardis, S.I.; Klapa, M.I.; Georgiou, C.D. Role of oxidative stress in Sclerotial differentiation and aflatoxin B1 biosynthesis in Aspergillus flavus. Appl. Environ. Microbiol. 2014, $80,5561-5571$.

11. Aguirre, J.; Rios-Momberg, M.; Hewitt, D.; Hansberg, W. Reactive oxygen species and development in microbial eukaryotes. Trends Microbiol. 2005, 13, 111-118.

12. Reverberi, M.; Zjalic, S.; Ricelli, A.; Fabbri, A.A.; Fanelli, C. Oxidant/antioxidant balance in Aspergillus parasiticus affects aflatoxin biosynthesis. Mycotoxin Res. 2006, 22, 39-47.

13. Chen, Q.; Vazquez, E.J.; Moghaddas, S.; Hoppel, C.L.; Lesnefsky, E.J. Production of reactive oxygen species by mitochondria: Central role of complex III. J. Biol. Chem. 2003, 278, 36027-36031.

14. Puntarulo, S.; Cederbaum, A.I. Production of reactive oxygen species by microsomes enriched in specific human cytochrome P450 enzymes. Free Radic. Biol. Med. 1998, 24, 1324-1330.

15. Skulachev, V.P. SkQ1 treatment and food restriction-Two ways to retard an aging program of organisms. Aging 2011, 3, 1045-1050.

16. Jiang, F.; Zhang, Y.; Dusting, G.J. NADPH oxidase-mediated redox signaling: Roles in cellular stress response, stress tolerance, and tissue repair. Pharmacol. Rev. 2011, 63, 218-242.

17. Montezano, A.C.; Touyz, R.M. Reactive oxygen species and endothelial function-Role of nitric oxide synthase uncoupling and Nox family nicotinamide adenine dinucleotide phosphate oxidases. Basic Clin. Pharmacol. Toxicol. 2012, 110, 87-94.

18. Cantu-Medellin, N.; Kelley, E.E. Xanthine oxidoreductase-catalyzed reactive species generation: A process in critical need of reevaluation. Redox Biol. 2013, 1, 353-358.

19. Lewis, D.F.; Sheridan, G. Cytochromes P450, oxygen, and evolution. Sci. World J. 2001, 1, 151-167.

20. Gessler, N.N.; Aver'yanov, A.A.; Belozerskaya, T.A. Reactive oxygen species in regulation of fungal development. Biochemistry 2007, 72, 1091-1109.

21. Bondy, S.C.; Naderi, S. Contribution of hepatic cytochrome P450 systems to the generation of reactive oxygen species. Biochem. Pharmacol. 1994, 48, 155-159.

22. Shimamoto, N. A pathophysiological role of cytochrome p450 involved in production of reactive oxygen species. Yakugaku Zasshi 2013, 133, 435-450.

23. Sun, X.; Ai, M.; Wang, Y.; Shen, S.; Gu, Y.; Jin, Y.; Zhou, Z.; Long, Y.; Yu, Q. Selective induction of tumor cell apoptosis by a novel P450-mediated reactive oxygen species (ROS) inducer methyl 3-(4-nitrophenyl) propiolate. J. Biol. Chem. 2013, 288, 8826-8837.

24. Chanda, A.; Roze, L.V.; Kang, S.; Artymovich, K.A.; Hicks, G.R.; Raikhel, N.V.; Calvo, A.M.; Linz, J.E. A key role for vesicles in fungal secondary metabolism. Proc. Natl. Acad. Sci. USA 2009, 106, 19533-19538.

25. Trail, F.; Mahanti, N.; Rarick, M.; Mehigh, R.; Liang, S.H.; Zhou, R.; Linz, J.E. Physical and transcriptional map of an aflatoxin gene cluster in Aspergillus parasiticus and functional disruption of a gene involved early in the aflatoxin pathway. Appl. Environ. Microbiol. 1995, 61, 2665-2673.

26. Narasaiah, K.V.; Sashidhar, R.B.; Subramanyam, C. Biochemical analysis of oxidative stress in the production of aflatoxin and its precursor intermediates. Mycopathologia 2006, 162, 179-189. 
27. Zeng, H.; Hatabayashi, H.; Nakagawa, H.; Cai, J.; Suzuki, R.; Sakuno, E.; Tanaka, T.; Ito, Y.; Ehrlich, K.C.; Nakajima, H.; et al. Conversion of 11-hydroxy-O-methylsterigmatocystin to aflatoxin G1 in Aspergillus parasiticus. Appl. Microbiol. Biotechnol. 2011, 90, 635-650.

28. Ehrlich, K.C.; Li, P.; Scharfenstein, L.; Chang, P.K. HypC, the anthrone oxidase involved in aflatoxin biosynthesis. Appl. Environ. Microbiol. 2010, 76, 3374-3377.

29. Cai, J.J.; Zeng, H.M.; Shima, Y.; Hatabayashi, H.; Nakagawa, H.; Ito, Y.; Adachi, Y.; Nakajima, H.; Yabe, K. Involvement of the nadA gene in formation of G-group aflatoxins in Aspergillus parasiticus. Fungal Genet. Biol. 2008, 45, 1081-1093.

30. Roze, L.V.; Arthur, A.E.; Hong, S.Y.; Chanda, A.; Linz, J.E. The initiation and pattern of spread of histone $\mathrm{H} 4$ acetylation parallel the order of transcriptional activation of genes in the aflatoxin cluster. Mol. Microbiol. 2007, 66, 713-726.

31. Yu, J.; Chang, P.; Bhatnagar, D.; Cleveland, T.E. Cloning of a sugar utilization gene cluster in Aspergillus parasiticus. Biochim. Biophys. Acta 2000, 1493, 211-214.

32. Price, M.S.; Yu, J.; Nierman, W.C.; Kim, H.S.; Pritchard, B.; Jacobus, C.A.; Bhatnagar, D.; Cleveland, T.E.; Payne, G.A. The aflatoxin pathway regulator AflR induces gene transcription inside and outside of the aflatoxin biosynthetic cluster. FEMS Microbiol. Lett. 2006, 255, 275-279.

33. Miyoshi, A.; Rochat, T.; Gratadoux, J.J.; Le Loir, Y.; Oliveira, S.C.; Langella, P.; Azevedo, V. Oxidative stress in Lactococcus lactis. Genet. Mol. Res. 2003, 2, 348-359.

34. Chanda, A.; Roze, L.V.; Linz, J.E. A possible role for exocytosis in aflatoxin export in Aspergillus parasiticus. Eukaryot. Cell 2010, 9, 1724-1727.

35. Chanda, A.; Roze, L.V.; Pastor, A.; Frame, M.K.; Linz, J.E. Purification of a vesicle-vacuole fraction functionally linked to aflatoxin synthesis in Aspergillus parasiticus. J. Microbiol. Methods 2009, 78, 28-33.

36. Towner, R.A.; Qian, S.Y.; Kadiiska, M.B.; Mason, R.P. In vivo identification of aflatoxin-induced free radicals in rat bile. Free Radic. Biol. Med. 2003, 35, 1330-1340.

37. Aslan, A.; Agar, G.; Alpsoy, L.; Kotan, E.; Ceker, S. Protective role of methanol extracts of two lichens on oxidative and genotoxic damage caused by AFB1 in human lymphocytes in vitro. Toxicol. Ind. Health 2012, 28, 505-512.

38. Rohlfs, M.; Albert, M.; Keller, N.P.; Kempken, F. Secondary chemicals protect mould from fungivory. Biol. Lett. 2007, 3, 523-525.

39. Doll, K.; Chatterjee, S.; Scheu, S.; Karlovsky, P.; Rohlfs, M. Fungal metabolic plasticity and sexual development mediate induced resistance to arthropod fungivory. Proc. Biol. Sci. 2013, 280, 1-8.

40. Edreva, A.; Velikova, V.; Tsonev, T.; Dagnon, S.; Gurel, A.; Aktas, L.; Gesheva, E. Stress-protective role of secondary metabolites: Diversity of functions and mechanisms. Gen. Appl. Plant Physiol. 2008, 34, 67-78.

41. Menke, J.; Weber, J.; Broz, K.; Kistler, H.C. Cellular development associated with induced mycotoxin synthesis in the filamentous fungus Fusarium graminearum. PLoS One 2013, 8, e63077.

42. Linz, J.E.; Chanda, A.; Hong, S.Y.; Whitten, D.A.; Wilkerson, C.; Roze, L.V. Proteomic and biochemical evidence support a role for transport vesicles and endosomes in stress response and secondary metabolism in Aspergillus parasiticus. J. Proteome Res. 2012, 11, 767-775. 
43. Mahanti, N.; Bhatnagar, D.; Cary, J.W.; Joubran, J.; Linz, J.E. Structure and function of fas-1A, a gene encoding a putative fatty acid synthetase directly involved in aflatoxin biosynthesis in Aspergillus parasiticus. Appl. Environ. Microbiol. 1996, 62, 191-195.

44. Keller, N.P.; Turner, G.; Bennett, J.W. Fungal secondary metabolism-From biochemistry to genomics. Nat. Rev. Microbiol. 2005, 3, 937-947.

45. Forseth, R.R.; Amaike, S.; Schwenk, D.; Affeldt, K.J.; Hoffmeister, D.; Schroeder, F.C.; Keller, N.P. Homologous NRPS-like gene clusters mediate redundant small-molecule biosynthesis in Aspergillus flavus. Angew. Chem. Int. Ed. Engl. 2013, 52, 1590-1594.

46. Rodriguez-Urra, A.B.; Jimenez, C.; Nieto, M.I.; Rodriguez, J.; Hayashi, H.; Ugalde, U. Signaling the induction of sporulation involves the interaction of two secondary metabolites in Aspergillus nidulans. ACS Chem. Biol. 2012, 7, 599-606.

47. Roze, L.V.; Hong, S.Y.; Linz, J.E. Aflatoxin biosynthesis: Current frontiers. Annu. Rev. Food Sci. Technol. 2013, 4, 293-311.

48. Chen, Z.H.; Yoshida, Y.; Saito, Y.; Niki, E. Adaptation to hydrogen peroxide enhances PC12 cell tolerance against oxidative damage. Neurosci. Lett. 2005, 383, 256-259.

49. Amin, V.M.; Olson, N.F. Selective increase in hydrogen peroxide resistance of a coagulase-positive Staphylococcus. J. Bacteriol. 1968, 95, 1604-1607.

50. Xu, F.J.; Jin, C.W.; Liu, W.J.; Zhang, Y.S.; Lin, X.Y. Pretreatment with $\mathrm{H}_{2} \mathrm{O}_{2}$ alleviates aluminum-induced oxidative stress in wheat seedlings. J. Integr. Plant Biol. 2011, 53, 44-53.

51. Guzel, S.; Terzi, R. Exogenous hydrogen peroxide increases dry matter production, mineral content and level of osmotic solutes in young maize leaves and alleviates deleterious effects of copper stress. Bot. Stud. 2013, 54, 26.

52. Calvo, A.M. The VeA regulatory system and its role in morphological and chemical development in fungi. Fungal Genet. Biol. 2008, 45, 1053-1061.

53. Varga, J.; Frisvad, J.C.; Samson, R.A. Two new aflatoxin producing species, and an overview of Aspergillus section Flavi. Stud. Mycol. 2011, 69, 57-80.

54. Zhou, R.; Linz, J.E. Enzymatic function of the nor-1 protein in aflatoxin biosynthesis in Aspergillus parasiticus. Appl. Environ. Microbiol. 1999, 65, 5639-5641.

55. Dutton, M.F. Enzymes and aflatoxin biosynthesis. Microbiol. Rev. 1988, 52, 274-295.

56. Amare, M.G.; Keller, N.P. Molecular mechanisms of Aspergillus flavus secondary metabolism and development. Fungal Genet. Biol. 2014, 66, 11-18.

57. Nelson, D.R. Progress in tracing the evolutionary paths of cytochrome P450. Biochim. Biophys. Acta 2011, 1814, 14-18.

(C) 2015 by the authors; licensee MDPI, Basel, Switzerland. This article is an open access article distributed under the terms and conditions of the Creative Commons Attribution license (http://creativecommons.org/licenses/by/4.0/). 